\title{
Emergência de respostas de ouvinte após ensino de intraverbais em um menino com Autismo
}

\section{Emergence of listener responses after intraverbal teaching in a boy with autism}

\section{Emergencia de respuestas de oyente después de la enseñanza de intraverbales en un niño con autismo}

\author{
Elaine de Carvalho Silva', Luiza Magalhães Caixeta ${ }^{2}$, Nassim Chamel Elias ${ }^{3}$
}

[1][2][3] Programa de Pós-Graduação em Educação Especial - Universidade Federal de São Carlos I Título abreviado: Relações intraverbais-ouvinte I Endereço para correspondência: Nassim Chamel Elias; e-mail: nchamel@terra.com.br; endereço: Departamento de Psicologia, Universidade Federal de São Carlos, Rod. Washington Luís, km 235 - SP-310 - São Carlos - SP, CEP 13565-905 I Email: rriques@gmail.coml doi: doi.org/10.18761/PAC.2020.v11.n2.03 


\begin{abstract}
The literature has indicated that interventions that use the principles of Applied Behavior Analysis have produced promising results for addressing language skill deficits presented by children with Autism Spectrum Disorder (ASD). Considering that the difficulties of communication impair the quality of life of these children, it is justified the teaching of speaker (expressive language) and listener (receptive language) repertoires. Since these repertoires are functionally independent, the present study used Multiple Exemplar Instruction procedures to promote interdependence of the teaching of intraverbal responses ("Which Hand Is This?" - answer: "Right") in the emergence of listener responses ("Show your right hand"). The objective was to verify the effects of teaching intraverbal responses of saying right or left for questions regarding the side of a body part (speaker repertoire) on the emergence of listener responses involving body parts (arms, hands, ears and legs) and spatial relationships of right and left in a six-year-old verbal boy with ASD. It was also verified the generalization of these relations to objects in the environment. The results indicated that the training of intraverbal responses prompted follow instruction listener responses to the participant, which confirms one of the teaching routes described in the literature (speaker-listener).
\end{abstract}

Keywords: Verbal behavior; Intraverbal; Listener behavior; Autism Spectrum Disorder.

Resumen: La literatura ha indicado que las intervenciones que utilizan los principios del análisis del comportamiento aplicado han producido resultados prometedores para solucionar problemas relacionados con los déficits en las habilidades de los lenguajes presentados por los niños con trastorno del espectro del autismo (TEA). Considerando que las dificultades de comunicación perjudican la calidad de vida de estos niños, se justifica la enseñanza de repertorios de hablante (lenguaje expresivo) y de oyente (lenguaje receptivo). En el presente estudio utilizó procedimientos de Instrucción con Múltiples Ejemplares para promover interdependencia de la enseñanza de respuestas intraverbales (“¿Qué mano es ésta?” - respuesta: "Derecha") en la emergencia de respuestas de oyente ("Muestre la mano derecha"). El objetivo fue verificar los efectos de la enseñanza de respuestas intraverbales de decir derecha o izquierda para preguntas sobre el lado de una parte del cuerpo en la emergencia de respuestas de oyente envolviendo partes del cuerpo (brazos, manos, orejas y piernas) y relaciones espaciales de derecha e izquierda en un niño verbal de seis años de edad con TEA. Se verificó también la generalización de esas relaciones para objetos en el ambiente. Los resultados indicaron que el entrenamiento de respuestas intraverbales promovió respuestas de oyente de seguir instrucción para el participante, lo que confirma una de las rutas de enseñanza descritas en la literatura (hablante-oyente).

Palabras clave: Comportamiento Verbal; Intraverbal; Comportamiento de oyente; Trastorno del Espectro del Autismo. 
Crianças diagnosticadas com Transtorno do Espectro Autista (TEA) apresentam, em graus variados, conforme o DSM-5 (APA, 2013), déficits na comunicação e interação social, além de comportamentos e interesses restritos e repetitivos. Esses déficits na comunicação podem ocorrer em graus variados e com topografias distintas, ou seja, existem diferenças nas habilidades que cada indivíduo tem de compartilhar informações com outros. Além disso, em alguns casos, o indivíduo não desenvolve a habilidade de se comunicar, seja por dificuldades de produzir fala (topografia) ou de utilizar a fala de forma adequada e contextualizada (função), ou desenvolve uma linguagem imatura, caracterizada por jargões, ecolalia, reversões de pronome, prosódia anormal, entonação monótona, entre outros (Gadia, Tuchman, \& Rotta, 2004). Crianças com TEA apresentam alguns sintomas que as diferenciam de outras crianças que apresentam apenas atraso na linguagem. Estes sintomas podem ser "perturbações da comunicação não-verbal, comportamentos estereotipados e perseverantes, interesses restritos e/ou incomuns e alterações das capacidades sociais" (Schirmer, Fontoura, \& Nunes, 2004, p. 98).

Em relação à aquisição da linguagem, Lowenkron (1998) afirma que crianças aprendem, primeiramente, repertórios receptivos, no qual respostas verbais de outras pessoas controlam determinadas respostas, sendo que esse repertório é uma indicação de compreensão verbal. Entretanto, conforme sugere Wilder (2011), uma preocupação comum envolvendo crianças e adultos com TEA é a falha em seguir um comando específico (como não seguir ou não compreender uma instrução de iniciar ou completar uma tarefa).

Skinner (1957) fala sobre a importância dos comportamentos precorrentes na comunicação, que são definidos como respostas que aumentam a probabilidade de outras respostas (correntes) ocorrerem ou serem reforçadas. Estas respostas podem ser estímulos especificadores de contingências que alteram a função do comportamento, facilitando ao ouvinte entrar em contato com as contingências descritas pelos operantes verbais (Simonassi \& Cameschi, 2003). Para aumentar a probabilidade que ocorra uma resposta em relação a uma instrução verbal é importante que o ouvinte tenha aprendido o comportamento solicitado. Portanto, para garantir que o ouvinte emita a resposta esperada, deve-se recorrer a todos os termos da contingência aos quais o comportamento solicitado possa estar funcionalmente relacionado (Alves \& Ribeiro, 2007; Medeiros \& Bernardes, 2009; Simonassi \& Cameschi, 2003). Um exemplo é a execução de movimentos (respostas motoras) que envolva a discriminação entre o lado direito e o lado esquerdo, sob controle de uma instrução apresentada por um falante.

Os estudos de Lamarre e Holland (1985), Alves e Ribeiro (2007), Medeiros e Bernardes (2009), Luke et al. (2011) e Silva e Elias (2018) investigaram a aquisição de operantes verbais utilizando relações espaciais do tipo direita/esquerda com procedimentos de instrução com múltiplos exemplares (MEI - do inglês Multiple Exemplar Instruction). O MEI pode ser usado de duas maneiras. Uma delas é como uma tática na qual diferentes formas de estímulos para o mesmo operante são apresentadas e rotacionadas durante o ensino (por exemplo, perna esquerda, orelha esquerda, mão esquerda; perna direita, orelha direita, mão direita) com a função de estabelecer classes funcionais ou conceitos. A outra maneira é como uma tática para tornar operantes inicialmente independentes em operantes que compartilham de um mesmo controle de estímulos através da rotação das respostas (respostas de tato e mando com mesma topografia) para um mesmo estímulo em um set instrucional (Eby, Greer, Tullo, Baker, \& Pauly 2010), como ensinar tatos (dizer o nome na presença de um estímulo não-verbal, que produziria reforço social) e, em seguida, mandos (pedir pelo mesmo item, que produziria acesso ao item) para os mesmos estímulos (por exemplo, itens comestíveis).

Silva e Elias (2018) tiveram por objetivo verificar os efeitos do ensino de respostas de ouvinte para relações espaciais de esquerda/direita envolvendo partes do corpo no uso dessas relações com objetos, em dois experimentos. No primeiro, foi utilizado um delineamento do tipo A-B com um menino com TEA e dois jovens com deficiência intelectual. No segundo, foi utilizado o delineamento de linha de base múltipla entre participantes com quatro meninos com TEA. O ensino foi realizado com MEI e com uso de esvanecimento de dicas de imitação, gestuais e físicas. Foram ensinadas ações que envol- 
viam partes do corpo e relações espaciais (Levante o braço direito/esquerdo, Toque a orelha direita/esquerda, Mostre a mão direita/esquerda, Levante a perna direita/esquerda) e foram testadas ações que envolviam objetos e relações espaciais (Sente na cadeira da direita/esquerda, Levante o cartão da direita/esquerda, Pegue o carrinho da direita/esquerda). O procedimento de ensino foi o mesmo nos dois experimentos e cada tentativa iniciava com o participante em pé, em frente à experimentadora; após obter a atenção do participante, a experimentadora fornecia uma instrução (por exemplo, "Levante o braço esquerdo"); respostas corretas foram seguidas de elogio verbal e acesso a trechos de vídeo apresentados por computador; respostas incorretas implicavam o fornecimento de dicas de imitação, ajudas físicas ou dicas gestuais na tentativa seguinte; instruções contendo os lados direito e esquerdo e partes distintas do corpo eram intercaladas em cada bloco de ensino. Os resultados indicaram aprendizagem das relações ensinadas para as partes do corpo e uso dessas relações para os objetos. Entretanto, foram necessárias aproximadamente 30 sessões para completar o procedimento no primeiro experimento e 45 no segundo experimento.

Neste contexto, um aspecto importante e que ainda constitui um assunto polêmico na área, segundo Petursdottir e Carr (2011), refere-se à sequência de ensino envolvendo repertórios expressivos (comportamento de falante) e receptivos (comportamento de ouvinte). Os autores sugerem que o ensino de repertórios de falante é mais eficaz na emergência de repertórios de ouvinte do que o ensino de repertórios de ouvinte na emergência de repertórios de falante (Bao, Sweatt, Lechago, \& Antal, 2017; Cuvo \& Riva, 1980; Wynn \& Smith, 2003). Protocolos expressivos são geralmente aqueles que têm por objetivo estabelecer respostas vocais da criança (ou alguma forma de comunicação alternativa) enquanto nos protocolos receptivos, a criança responde de forma não-verbal a instruções verbais (Petursdottir \& Carr, 2011).

Os estudos encontrados que ensinaram relações espaciais (Alves \& Ribeiro, 2007; Lamarre \& Holland, 1985; Lee, 1981; Luke et al., 2011; Medeiros \& Bernardes, 2009) dentro da Análise do Comportamento não utilizaram os conceitos de direita/esquerda para partes do corpo. A exceção foi o estudo de Silva e Elias (2018), que ensinaram e testaram somente relações de ouvinte, e, juntamente com Luke et al. (2011), foram os estudos que tiveram participantes com desenvolvimento atípico. Esse estudo pretendeu, portanto, expandir dados da literatura sobre os efeitos do ensino de respostas de falante na emergência de respostas de ouvinte com o uso do MEI e relações espaciais para um participante com TEA. Vale lembrar, como mencionado anteriormente, que indivíduos com TEA podem apresentar atrasos no desenvolvimento da linguagem e déficits em seguir comandos e instruções. Além disso, os conceitos de esquerda e direita são úteis em várias situações do dia-a-dia, como para participação social, locomoção, direcionamento e segurança.

Portanto, considerando a possibilidade apontada na literatura de que o treino de respostas verbais de falante pode aumentar a probabilidade da emergência de respostas de ouvinte, o presente estudo verificou os efeitos do ensino de respostas intraverbais de dizer direita ou esquerda para questões referentes ao lado de uma parte do corpo (repertório de falante) na emergência de respostas de ouvinte envolvendo partes do corpo (braços, mãos, orelhas e pernas) e relações espaciais de direita e esquerda. Foi verificada também a generalização dessas relações para objetos no ambiente.

\section{Método}

Participante. O participante deste estudo foi um menino de seis anos de idade diagnosticado com TEA. Não era alfabetizado, não lia e não escrevia, mas suas respostas vocais (fala) eram corretas e seguiam as regras gramaticais, sendo que ele já apresentava em seu repertório respostas ecoicas, de mando e de tato e estava em aquisição de respostas intraverbais. Ocasionalmente, apresentava comportamento de choro para situações que ele não compreendia (geralmente em contextos sociais com muito barulho e gritos, segundo sua mãe) ou não sabia como emitir as respostas. O primeiro critério de inclusão foi de $80 \%$ ou mais de respostas corretas nos testes de repertório de imitação motora e de imitação vocal (descritas a seguir). O segundo critério foi a obtenção de, no máximo, $40 \%$ de respostas corretas nos 
pré-testes de respostas intraverbais, que envolviam questões relacionadas às partes do corpo e à relação espacial (por exemplo, dizer "direita ou esquerda" para a pergunta "Que mão é esta"?), e nos pré-testes de respostas de ouvinte (por exemplo, "mostrar a mão esquerda" quando esta ação fosse solicitada). O participante obteve $100 \%$ de respostas corretas nos testes de imitação motora e de imitação vocal; os resultados dos pré-testes de intraverbais e de ouvinte são apresentados na Figura 1.

Local. O presente estudo foi realizado na sala de jantar da residência da criança, onde havia apenas uma mesa grande retangular (para seis pessoas) e seis cadeiras. Sobre a mesa, eram colocados os materiais do estudo: os protocolos, lápis, um notebook (DELL - Inspiron 14) e um celular (Samsung Galaxy A5/2017 modelo SM-A520F) utilizado para apresentação de jogos que serviram como consequências para respostas corretas.

Delineamento Experimental e Sequência das Condições Experimentais. Foi utilizado o delineamento de pré e pós-teste tendo o sujeito como seu próprio controle (Cozby, 2003). A Tabela 1 apresenta a sequência das condições experimentais.

Tabela 1. Sequência das condições experimentais.

\begin{tabular}{ll}
\hline Condição Experimental & Critério \\
\hline Teste de imitação motora & Pelo menos $80 \%$ de respostas corretas \\
$\begin{array}{l}\text { Teste de imitação vocal } \\
\text { Avaliação de Preferência }\end{array}$ & Pelo menos $80 \%$ de respostas corretas \\
Teste de Repertório Receptivo & No máximo $40 \%$ de respostas corretas \\
Teste de Repertório Expressivo & No máximo $40 \%$ de respostas corretas \\
Ensino de Repertório Expressivo & Pelo menos $88 \%$ de respostas corretas \\
Pós-teste de Repertório Receptivo & \\
\hline
\end{tabular}

\section{Procedimento Experimental}

Teste de imitação motora. Esse teste foi composto de dez tentativas discretas que iniciaram com a instrução verbal "Faça isso" seguida de um movimento executado pela experimentadora: tocar o nariz, bater palmas, levantar os braços, tocar as orelhas, sentar-se na cadeira, levantar uma perna e outra alternando, olhar para um lado e para outro virando só a cabeça, colocar as mãos nos joelhos, levantar-se da cadeira, andar em volta da cadeira. Uma resposta era considerada correta quando o participante imitava exatamente o movimento feito pela pesquisadora. Respostas corretas eram seguidas de elogio e respostas incorretas eram seguidas da apresentação da próxima tentativa.

Teste de imitação vocal. Esse teste foi composto de dez tentativas discretas (DTT) que iniciaram com a instrução verbal "Diga" seguida de palavras ou pseudo-palavras distintas: cano, vala, pora, bero, difo, papiro, mesada, pintura, benego, vicajo Para a seleção destas palavras foram adotados dois critérios: (i) palavras com duas ou três sílabas e (ii) palavras com e sem significado para garantir imitação vocal generalizada. Uma resposta era considerada correta quando o participante dizia a mesma palavra que a experimentadora, de forma clara, com correspondência ponto-a-ponto. Respostas corretas eram seguidas de elogio e respostas incorretas eram seguidas da apresentação da próxima tentativa.

Avaliação de Preferência. Jogos para celular de preferência do participante foram indicados por sua mãe e apresentados no início de cada dia de sessão experimental para que ele pudesse escolher (Teste de Preferência com Múltiplos Estímulos sem Reposição; Carr, Nicolson, \& Higbee, 2000). Os ícones dos jogos eram abertos no tablet ou celular e o participante apontava para um deles. O jogo es- 
colhido era utilizado como consequência para cada resposta correta nas sessões daquele dia.

Teste de Repertório Receptivo. A experimentadora forneceu uma instrução identificando uma ação (que podia envolver um objeto ou parte do corpo) e a relação espacial ("Levante o braço esquerdo", "Toque a orelha direita", "Levante a perna direita", "Toque a orelha esquerda", "Levante o braço direito", "Levante a perna esquerda", "Sente na cadeira da esquerda", "Pegue o carrinho da esquerda", "Sente na cadeira da direita", "Pegue o carrinho da direita"). Respostas foram consideradas corretas se o participante realizasse a ação solicitada em até 10 s. Outras respostas foram consideradas incorretas. Estas instruções foram apresentadas randomicamente, de maneira que a mesma parte do corpo ou o mesmo objeto e a mesma relação espacial não fossem apresentados em tentativas consecutivas. Não havia consequências diferenciais para respostas corretas ou incorretas.

Teste de Repertório Expressivo. A experimentadora apresentou algumas perguntas, descritas a seguir, que tivessem como possíveis respostas "esquerda" ou "direita". As perguntas eram relacionadas a objetos ou partes do corpo do participante. Respostas foram consideradas corretas se o participante dissesse a relação espacial correta em até 10s. Outras respostas foram consideradas incorretas. Não houve consequências diferenciais para respostas corretas e incorretas. Foram apresentadas as seguintes perguntas: "Que braço é esse?", "Que perna é essa?", "Que orelha é essa?", "Que mão é esta?", "De que lado está o carrinho?", "De que lado está a cadeira?". Enquanto apresentava cada pergunta a pesquisadora apontava o objeto ou a parte do corpo a que se referia. Essas instruções foram apresentadas randomicamente, de maneira que a mesma parte do corpo ou o mesmo objeto e a mesma relação espacial não fossem apresentados em tentativas consecutivas.

Ensino de Repertório Expressivo. A resposta esperada do participante era dizer direita ou esquerda após a apresentação de uma pergunta (intraverbal) em relação a uma parte do corpo. Cada tentativa de ensino foi iniciada com o participante em frente à experimentadora. Após obter a atenção do participante, a experimentadora apresentava uma pergunta (por exemplo, "Que braço é este?"), enquanto apontava para a parte do corpo do participante. Respostas foram consideradas corretas quando o participante disse o lado correspondente em até 10s e foram seguidas de elogio verbal ("Parabéns!", "Muito bem!", "Isso mesmo!") e da apresentação do jogo de celular escolhido naquele dia por um tempo de 20 a 30 segundos. Este procedimento foi realizado após cada resposta correta. Outras respostas foram consideradas incorretas. Respostas incorretas foram seguidas de procedimento de correção com fornecimento de dicas de imitação vocal na tentativa seguinte (por exemplo, ao perguntar "Que braço é esse?" e apontar para o braço esquerdo do participante, a resposta correta foi dada imediatamente "Esquerdo" para o participante repetir/ ecoar). O esvanecimento de dicas foi realizado em três etapas, sempre que havia uma resposta incorreta: (1) foi fornecido o modelo imediato (palavra) completo na primeira tentativa de correção, logo em seguida da imitação vocal do participante, (2) mesmo com a imitação correta na primeira tentativa, na tentativa seguinte, era fornecido o modelo parcial imediato (apenas a primeira silaba da palavra) e, por último (3) a segunda etapa era repetida, porém, num volume de voz mais baixo. Assim, a solicitação era reapresentada até que o participante pudesse emitir uma resposta independente (sem dica). Na sequência, era introduzido o mesmo procedimento para outra pergunta. Após o participante atingir o critério para cada pergunta separadamente, as perguntas eram apresentadas de forma randomizada e intercalada até que o participante atingisse o critério de pelo menos oito respostas corretas independentes em nove tentativas consecutivas (88\% de respostas corretas). Foram apresentadas as seguintes perguntas: "Que braço é esse?", "Que perna é essa?", "Que orelha é essa?", "Que mão é essa?". Portanto, foram ensinados múltiplos exemplares para esquerda e direita (diferentes formas do mesmo estímulo foram apresentadas e rotacionadas durante o ensino; Eby, Greer, Tullo, Baker \& Pauly 2010). Não houve um número especifico de tentativas para cada sessão, que eram apresentadas até alcance de critério ou final do período da sessão. 
Pós-teste de Repertório Receptivo. A experimentadora forneceu uma instrução identificando uma ação que envolvia uma parte do corpo e a relação espacial ("Levante o braço esquerdo", "Toque a orelha direita", "Levante a perna direita", "Toque a orelha esquerda", "Levante o braço direito", "Levante a perna esquerda"). Respostas foram consideradas corretas se o participante realizasse a ação solicitada em até 10s. Outras respostas foram consideradas incorretas. Essas instruções foram apresentadas randomicamente, de maneira que a mesma parte do corpo e mesma relação espacial não fossem apresentadas em tentativas consecutivas. Não havia consequências diferenciais para respostas corretas ou incorretas.

Pós-Teste de Generalização. A experimentadora forneceu uma instrução identificando uma ação que envolvia um objeto e a relação espacial ("Sente na cadeira da esquerda", "Pegue o carrinho da esquerda", "Sente na cadeira da direita", "Pegue o carrinho da direita"). Nas tentativas com cadeiras, o participante era posicionado entre duas cadeiras idênticas antes da instrução ser fornecida; nas tentativas com carrinhos, dois carrinhos idênticos eram colocados sobre uma mesa, em frente ao participante, antes da instrução ser fornecida. Respostas foram consideradas corretas se o participante realizasse a ação solicitada em até 10s. Outras respostas foram consideradas incorretas. Essas instruções foram apresentadas randomicamente, de maneira que o mesmo objeto e mesma relação espacial não fossem apresentados em tentativas consecutivas. Não havia consequências diferenciais para respostas corretas ou incorretas.

Análise de concordância dos dados. Todas as sessões foram filmadas para registro dos dados pela experimentadora e por um segundo observador para cálculos de fidedignidade. A concordância foi calculada dividindo-se o número de concordâncias pelo número de concordâncias mais o número de discordâncias e multiplicado por 100. Houve concordância de 100\%.

Análise do procedimento. Foi verificada consistência procedural, em que o comportamento da experimentadora na aplicação correta do procedimento foi avaliado por um observador que preencheu uma ficha com o registro desses comportamentos, em $40 \%$ das filmagens. A ficha era preenchida para cada tentativa, indicando se houve apresentação correta da instrução, apresentação correta dos materiais, ausência de dicas não programadas e intervalo entre as tentativas. Os resultados indicaram que a experimentadora realizou $90 \%$ das tentativas de acordo com o procedimento.

Aspectos éticos da pesquisa. De acordo com as diretrizes e normas que regem a pesquisa científica, este estudo foi submetido ao Comitê de Ética em Pesquisas em Seres Humanos. Após a obtenção da aprovação do comitê (CAAE: 76495417.9.0000.5504), o trabalho de pesquisa, com seus objetivos e procedimentos, foi apresentado aos pais do participante, para que, caso concordassem com a participação de seu filho, fizessem a leitura e assinatura do Termo de Consentimento Livre e Esclarecido, para efetivar a autorização.

\section{Resultados}

A Figura 1 mostra o desempenho do participante nos testes de repertório receptivo e expressivo em linha de base, sessões de ensino e pós-teste. O eixo vertical (eixo Y) mostra a porcentagem de acertos em cada sessão. O eixo horizontal (eixo X) é referente às sessões de linha de base (sessões de 1 a 3 ), de ensino (sessões de 4 a 6) e pós-teste (sessões 7 e 8 ).

A porcentagem de acertos nos testes iniciais de repertório receptivo e expressivo em linha de base ficou abaixo de $40 \%$ em três sessões consecutivas. O participante alcançou o critério de aprendizagem de $88 \%$ de respostas corretas em cento e nove tentativas num total de três sessões, sendo que na primeira sessão de ensino já houve aumento no desempenho no repertório expressivo (que variou de 14 a $34 \%$ na linha de base para $49 \%$ na primeira sessão de ensino). No pós-teste, o participante apresentou $75 \%$ de respostas corretas para as partes do corpo e $67 \%$ no teste de generalização para os objetos. Esses dados indicam melhora de uma média de $26 \%$ de acertos em linha de base para $71 \%$ no pós-teste para os repertórios de ouvinte, considerando o desempenho para as relações que envolviam partes do corpo e objetos. 


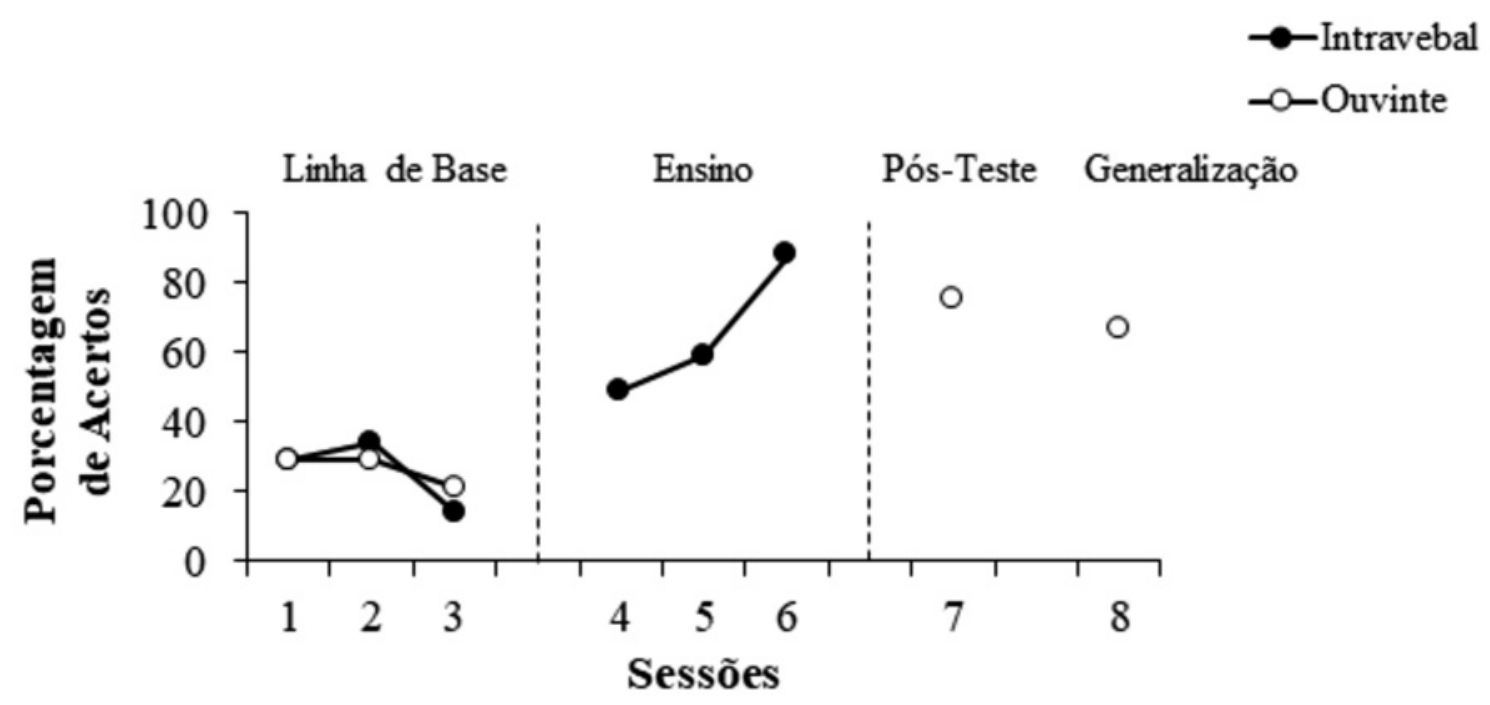

Figura 1. Desempenho do participante ao longo do procedimento.

\section{Discussão}

O presente estudo teve como objetivo verificar os efeitos do ensino de respostas intraverbais de dizer direita ou esquerda para questões referentes ao lado de uma parte do corpo na emergência de respostas de ouvinte envolvendo partes do corpo e relações espaciais de direita e esquerda, assim como verificar a generalização para objetos. A melhora de desempenho observada nos pós-testes em relação à linha de base corrobora a afirmação de Petursdottir e Carr (2011) de que o ensino de repertórios de falante (ou expressivos) é eficaz na emergência de repertórios de ouvinte (ou receptivos).

Nessa mesma linha, os resultados de Silva e Elias (2018) sugerem que novos comportamentos de ouvinte emergem após ensino de outros comportamentos de ouvinte. Entretanto, o número de sessões para alcance de critério (de 30 a 45 sessões) foi muito maior do que no presente estudo (apenas três sessões). Esses dados sugerem, novamente, que o ensino de relações de falante é mais eficaz na emergência de repertórios de ouvinte. Entretanto, o menor número de sessões para alcance de critério do participante do presente estudo pode estar relacionado com seu repertório verbal prévio, que era mais desenvolvido do que o dos participantes de Silva e Elias (2018).
Os resultados promissores desse estudo também podem ser interpretados a partir da Teoria da Nomeação, de Horne e Lowe (1996), que propõe a nomeação como um operante de ordem superior definida como uma relação bidirecional entre um componente do falante e um do ouvinte e ocorre quando apenas um desses dois componentes é suficiente para estabelecer ambas as relações. Dessa forma, pode-se inferir que o participante dessa pesquisa já apresentava relações de nomeação para outros estímulos. Portanto, como sugerem Elias e Arantes (2019), o desenvolvimento da nomeação parece ser um pré-requisito importante para o desenvolvimento de novas formas de linguagem.

Uma questão sugerida por Wilder (2011) refere-se às possíveis dificuldades de uma criança com TEA em compreender uma solicitação ou uma instrução no sentido de realizar uma ação ou uma tarefa qualquer. Os resultados do presente estudo poderiam ser expandidos para outras situações do cotidiano, como responder a instruções do tipo "Entre na porta do lado direito"; "Olhe para o lado esquerdo ao atravessar a rua", "De que lado está doendo?", “Qual é a perna machucada?", etc.

Os resultados desse estudo expandem o que já havia sido documentado na literatura (Alves \& Ribeiro, 2007; Silva \& Elias, 2018; Lamarre \& Holland, 1985; Lee, 1981; Luke et al., 2011; Medeiros \& Bernardes, 
2009) dentro da Análise do Comportamento em relação ao aprendizado de relações espaciais de direita/ esquerda para partes do corpo para relações de ouvinte e relações intraverbais.

Neste contexto, o procedimento de MEI relacionado às partes do corpo e relações espaciais (lados direito e esquerdo) foi uma estratégia eficaz para aquisição de respostas intraverbais e emergência de respostas de ouvinte para as relações ensinadas (partes do corpo) e para novas relações (generalização para objetos do ambiente). Pode-se inferir que o treino intraverbal aliado ao procedimento de MEI promoveu respostas de ouvinte para o participante, o que confirma uma das rotas de ensino descritas na literatura. Porém, outros estudos poderiam replicar o procedimento com um número maior de participantes e com outros repertórios de entrada. Adicionalmente, poderia ser conduzido um estudo em que fossem comparados, diretamente, os efeitos do treino de respostas intraverbais na emergência de respostas de ouvinte com os efeitos do treino de respostas de ouvinte na emergência de respostas intraverbais para partes do corpo e relações espaciais em crianças com TEA.

Este estudo ofereceu uma dimensão concreta para o repertório receptivo e expressivo ao utilizar as partes do corpo e duas relações espaciais como estratégia de ensino, pois, na maioria dos estudos encontrados foram utilizados outros materiais (figuras ou símbolos digitais). De acordo com Gallahue e Ozmun (2005), a estratégia corporal pode favorecer outras áreas de desenvolvimento, como a psicomotora e a cognitiva, visto que pode haver atraso no desenvolvimento motor desta população e esta possibilidade prejudica a noção espacial, o que consequentemente interfere na interação do indivíduo com o meio ambiente afetando, inclusive, a linguagem. Portanto, é possível que estímulos relacionados à noção do próprio corpo possam favorecer a elaboração da linguagem de indivíduos inseridos no TEA.

\section{Referências}

Alves, C. \& Ribeiro, A. F. (2007). Relações entre tatos e mandos durante a aquisição. Revista Brasileira de Terapia Comportamental e Cognitiva, 9(2), 289-305.

American Psychiatric Association (APA) (2013). Diagnostic and Statistical Manual of Mental Disorders: DSM-V, 5th ed. Arlington, VA.

Bao, S., Sweatt, K. T., Lechago, S. A., \& Antal, S. (2017). The effects of receptive and expressive instructional sequences on varied conditional discriminations. Journal of Applied Behavior Analysis, 50, 775- 788.

Carr, J. E., Nicolson, A. C., \& Higbee, T. S. (2000). Evaluation of a brief multiple-stimulus preference assessment in a naturalistic context. Journal of Applied Behavior Analysis, 33(3), 353-357.

Cozby, P. C. (2003). Métodos de pesquisa em ciências do comportamento. Editora Atlas S.A. São Paulo.

Cuvo, A. J. \& Riva, M. T. (1980). Generalization and transfer between comprehension and production: A comparison of retarded and nonretarded persons. Journal of Applied Behavior Analysis, 13, 315-331.

Eby, C. M., Greer, R. D., Tullo, L. D., Baker, K. A., \& Pauly, R. (2010). Effects of Multiple Exemplar Instruction on the transformation of stimulus function across written and vocal spelling instruction responses by students with autism. The Journal of Speech-Language Pathology and Applied Behavior Analysis, 5, 20-31.

Elias, N. C. \& Arantes, A. (2019). Teorias comportamentais sobre a etiologia do autismo e uma nova proposta. In J. C. Luzia, J. Gamba, N. Kienen, \& S. R. de S. A. Gil (eds), Psicologia e análise do comportamento: pesquisa e intervenção ( $1^{\text {a }}$ ed). Londrina: Universidade Estadual de Londrina, 1, 134-146.

Gadia, C. A., Tuchman, R., \& Rotta, N. T. (2004). Autismo e doenças invasivas de desenvolvimento. Jornal de Pediatria, 80(2, Suppl.), 83-94.

Gallahue, D. L., \& Ozmun, J. C. (2005). Compreendendo o desenvolvimento motor: bebês, crianças, adolescentes e adultos. São Paulo: Phorte. 
Horne, P. J., \& Lowe, C. F. (1996). On the origins of naming and other symbolic behavior. Journal of the Experimental Analysis of Behavior, 65, 185-241.

Lamarre, J. \& Holland, J. G. (1985). The functional independence of mands and tacts. Journal of the Experimental Analysis of Behavior, 43(1), 5-19.

Lee, V. L. (1981). Prepositional phrases spoken and heard. Journal of the Experimental Analysis of Behavior, 35(2), 227-242.

Lowenkron, B. (1998). Some logical functions of joint control. Journal of the Experimental Analysis of Behavior, 69, 327-354.

Luke, N., Greer, R. D., Singer-Dudek, J., \& Keohane, D. (2011). The Emergence of autoclitic frames in atypically and typically developing children as a function of Multiple Exemplar Instruction. The Analysis of Verbal Behavior, 27, 141-156.

Medeiros, C. A. \& Bernardes, M. C. (2009). Estabelecimento de repertório de transposição entre mandos e tatos durante a aquisição de nomes de posições. Revista Brasileira de Análise do Comportamento, 5(2), 51-68.

Petursdottir, A. I. \& Carr, J. E. (2011). A review of recommendations for sequencing receptive and expressive language instruction. Journal of Applied Behavior Analysis, 44(4), 859-876.

Schirmer, C. R., Fontoura, D. R., \& Nunes, M. L. (2004). Distúrbios da aquisição da linguagem e da aprendizagem. Journal da Pediatria, 80(2), 95-103.

Silva, E. C. \& Elias, N. C. (2018). Ensino de relações espaciais de esquerda e direita a participantes com autismo e deficiência intelectual. Psicologia: Teoria e Pesquisa, 33, 1-8.

Simonassi, R. E. \& Cameschi, C. E. (2003). O episódio verbal e a análise de comportamentos verbais privados. Revista Brasileira de Terapia Comportamental e Cognitiva, 5(2), 105-119.

Skinner, B. F. (1957). Verbal Behavior. New York: Applenton-Century-Crofts.

Wilder, D. (2011). Noncompliance and oppositional behavior. In Luiselli, J. K. (org), Teaching and behavior support for children and adults with Autism Spectrum Disorder: A practitioner's guide. New York: Oxford University Press.

Wynn, J. W. \& Smith, T. (2003). Generalization between receptive and expressive language in young children with autism. Behavioral Interventions, 18, 245-266.

Yin, R.K. (2005). Estudo de caso: planejamento e métodos. 3. ed. Porto Alegre: Bookman, 2005.

\section{Informações do Artigo}

Histórico do artigo:

Submetido em:15/05/2019

Primeira decisão editorial: 21/08/2020

Aceito em: 08/09/2020

Editor associado: Adriano Alves Barboza 